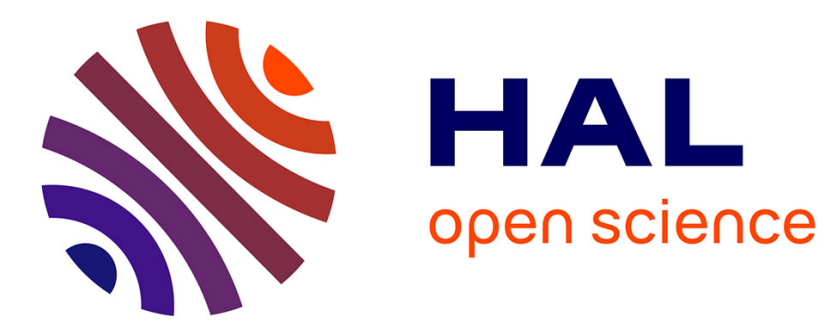

\title{
Les événements off: de la résistance à la mise en scène de la ville créative
}

Elsa Vivant

\section{To cite this version:}

Elsa Vivant. Les événements off: de la résistance à la mise en scène de la ville créative. Géocarrefour - Revue de géographie de Lyon, 2007, 82 (3), pp.131-140. halshs-00282122

\section{HAL Id: halshs-00282122 \\ https://shs.hal.science/halshs-00282122}

Submitted on 19 Nov 2008

HAL is a multi-disciplinary open access archive for the deposit and dissemination of scientific research documents, whether they are published or not. The documents may come from teaching and research institutions in France or abroad, or from public or private research centers.
L'archive ouverte pluridisciplinaire HAL, est destinée au dépôt et à la diffusion de documents scientifiques de niveau recherche, publiés ou non, émanant des établissements d'enseignement et de recherche français ou étrangers, des laboratoires publics ou privés. 


\section{Les événements off : de la résistance à la mise en scène de la ville créative}

Article publié en 2007 dans la revue Géocarrefour, vol. 82, n³.

\section{Elsa Vivant}

Maitre de Conférences

Laboratoire Théories des Mutations Urbaines - Institut Français d’Urbanisme

Université Paris 8

4 rue Nobel - Cité Descartes

77420 Champs sur Marne

Elsa.vivant@univ-paris8.fr

\section{Résumé :}

L’objet de cet article est de s'intéresser à des événements, conçus et produits en dehors des sphères institutionnelles ou commerciales, que nous appelons des événements off. Dans quelle mesure les scènes artistiques off peuvent-elles être des acteurs de la ville événementielle ? Les scènes off, par les spectacles, fêtes, soirées et événements qu'elles organisent, animent les villes. Elles deviennent même des acteurs incontournables de la vie nocturne urbaine. Mais l'événement peut avoir d'autres finalités que le plaisir de la fête et de la découverte artistique. Les scènes off utilisent le happening artistique comme un outil de communication à des fins diverses : faire connaitre son travail, accéder aux mondes de l'art, alerter le public sur ses difficultés, ... Les scènes artistiques off participent à la production sociale des représentations de la ville, au point parfois de devenir un élément essentiel de l'image de la ville. D’ailleurs, les pouvoirs publics locaux intègrent les scènes et événements off dans le cadre de stratégies plus larges de production de l'image de leur ville, fondée sur des représentations liées ou évoquant la créativité, l'innovation, la différence et la tolérance.

Mots-clés : scènes artistiques off, squats d’artistes, occupations artistiques, Paris, créativité.

\section{Abstract :}

This paper focuses on off events which are defined and implemented out of the institutional and commercial worlds of events. To what extend do off artistic scenes play a role in the city of events? Off scenes through shows, plays, events, parties and fiestas are part of the living city. They even become major actors of cities' nightlife. Events can also be used by off artists as tool for communication as much as local authorities are using events to promote their city. Thus, off events are opportunities for artists to claim for space, to search for artistic legitimacy and to look for customers. Off scenes are experimented by a more diverse audience. The local authorities are considering them as useful tool for communication to symbolize and to celebrate diversity, creativity, tolerance and openness.

Keywords : off artistic scenes, artistic squat, Paris, creativity. 


\section{Les événements off : de la résistance à la mise en scène de la ville créative}

Jeux Olympiques, Capitale européenne de la culture, exposition internationale, biennale d'art contemporain, féria, foire commerciale... La notion de ville événementielle est le plus souvent considérée sous l'angle des grands événements in organisés et mis en œuvre par les municipalités pour animer la ville. Au-delà de l'animation et des expériences singulières qu’ils créent, ces événements sont souvent des instruments des politiques municipales pour mettre en scène leur projet urbain (Masboungi, Gravelaine (de), 2004). Mais la ville événementielle n’est pas bornée par l'action publique ni par les intérêts des acteurs privés et commerciaux ${ }^{1}$. Dans une perspective plus large, la ville événementielle correspond à ces moments qui sortent la ville, ses habitants et ses visiteurs de leur routine et de leurs habitudes quotidiennes. Rituel saisonnier ou happening unique, l'événement urbain correspond à un usage de l'espace différent de ce pour lequel il avait été conçu. Ce détournement de l'espace est limité dans le temps. L'extraordinaireté de l'événement prend des formes et des usages très divers : de la mise en scène du paysage urbain par des illuminations hivernales à la reconquête des terrains vagues par des jardiniers du dimanche. L'appropriation collective et temporaire de l'espace public par des collectifs artistiques, l'organisation de fêtes aux marges de la légalité, le détournement de l'environnement urbain quotidien par des installations artistiques non-autorisées sont autant d'événements qui participent à l'animation de la ville, voire qui permettent sa mise en scène et produisent un nouveau discours et de nouvelles représentations sociales de l'espace. Ainsi, l'objet de cet article est de s'intéresser à des événements, conçus et produits en dehors des sphères institutionnelles ou commerciales, que nous appelons des événements off. Plus précisément, il s’agit de mettre en évidence dans quelle mesure les scènes artistiques off peuvent être des acteurs de la ville événementielle.

Les scènes off, par les spectacles, fêtes, soirées et événements qu'elles organisent, animent les villes. Elles deviennent même des acteurs incontournables de la vie nocturne urbaine. Mais l'événement peut avoir d'autres finalités que le plaisir de la fête et de la découverte artistique. Tout comme des municipalités mettent en scène leur projets par les événements, les scènes off utilisent le happening artistique comme un outil de communication à des fins diverses : se faire connaitre, faire connaitre et reconnaitre son travail, accéder aux mondes de l'art, alerter le public sur ses difficultés, informer sur ses projets... Par ces événements, les scènes artistiques off

\footnotetext{
${ }^{1}$ La plupart des événements publics sont sponsorisés, à des degrés divers, par des entreprises privées. Mais certaines entreprises, liées ou non au monde de la production et de la consommation culturelle, investissent temporairement l'espace public par l'organisation d'événements en plein air (comme les tournées musicales estivales de la société Ricard).
} 
prennent une place plus importante dans l'espace public. Elles participent à la production sociale des représentations de la ville. Au point parfois de devenir un élément essentiel de l’image de la ville, une attraction pour les visiteurs au même titre que les musées ou les festivals. D’ailleurs, les pouvoirs publics locaux prennent progressivement conscience de ce potentiel. Ils intègrent les scènes et événements off dans le cadre de stratégies plus larges de production de l'image de leur ville, fondée sur des représentations liées ou évoquant la créativité, l'innovation, la différence et la tolérance. La référence aux scènes artistiques off contemporaines est mobilisée dans la production d'un discours de la Ville sur la ville. Cette évolution de l'usage de l'événement par les scènes artistiques off vers leur instrumentalisation par les municipalités sera illustrée principalement par l'étude d'exemples parisiens, comme les Frigos dans le $13^{\text {ème }}$ arrondissement ou les mouvements de squats d'artistes ${ }^{2}$, mais aussi par quelques escapades dans d'autres villes européennes.

\section{Le off dans le système de la création artistique : espace de liberté, symbole de créativité}

Depuis une trentaine d'années, en France et ailleurs, se développent des expériences artistiques alternatives initiées par des acteurs associatifs, culturels ou artistiques, sans soutien public ou commercial. Ces pratiques, que nous appelons off, ont en commun d'être peu ou pas prises en compte par l'institution culturelle et de ne pas avoir une place claire dans le marché des biens culturels. Les scènes off appartiennent aux mondes de l'art tout en proposant une démarche artistique alternative au in, sphère de légitimation et de reconnaissance, qui puise continuellement inspiration et nouveaux talents dans le off. Elles sont souvent marquées par une très grande précarité économique, matérielle, et juridique. Elles peuvent être à la fois des pratiques innovantes à audience confidentielle ou des phénomènes de masse émergents, dans le cadre de la légalité ou non, isolés ou collectifs. Elles s’appuient sur des nouveaux supports (comme la vidéo) ou des pratiques (l'art dans la rue, l'art de la rue), comme par exemple les raves techno, le théâtre de rue, le graff', le hip-hop, le slam, les squats d'artistes, le rock alternatif, etc. Cette liste n'est ni exhaustive ni exclusive laissant la porte ouverte aux innovations et expérimentations nouvelles et évitant le cadre contraignant et restrictif d’une définition stricte (Vivant, 2006b).

L’émergence de ces pratiques culturelles off est à prendre en compte dans le cadre plus général de l'évolution de la création culturelle, caractérisée par l'évolution des codes esthétiques traditionnels, par le mélange des formes et des genres culturels, et la reconnaissance

\footnotetext{
${ }^{2}$ Ces exemples ont été l'objet d'enquêtes par entretiens auprès des acteurs, complétés par des visites de site, des observations, la consultation d'archives et la constitution de revues de presse (Vivant, 2006b).
} 
institutionnelle des sous-cultures. Dans le même temps, les pratiques culturelles sont marquées par la transcendance des divisions entre grande et basse culture (ou entre culture légitime et culture populaire) où l'éclectisme et l' « omnivorisme » sont les nouveaux modes de distinction et de légitimation culturelle ; l'individu hypermoderne construisant ses modes de consommation et ses goûts culturels au gré d'influences diverses (Ascher, 2001; Ascher, 2006 ; Donnat, 2004; Peterson, 2004).

\section{Le lieu off comme événement en soi}

Les pratiques et acteurs du off utilisent de manière plus ou moins temporaire des lieux divers qui sont à la fois des lieux off de la culture et les lieux de la culture off. De Marseille à Grenoble, de Berlin à Ljubljana, la création d'un lieu off résulte de la volonté d'un groupe qui souhaite défendre sa vision de la culture, ses pratiques et goûts culturels, c'est-à-dire des genres musicaux assez marginaux (ou portant une image de marginalité, comme le punk). Le désir d'expérimenter des modes de vie différents est aussi un des motifs de la création de certains lieux où l'organisation communautaire, l'autogestion, l'autosuffisance, les convictions écologiques et végétariennes sont les éléments d'un projet social et politique off qui s’articule avec le projet artistique off (Lextrait, 2001 ; TransEuropeHalles, 2001; Raffin, 2002). Les délaissés urbains, terrains vagues, squats, hangars désaffectés, friches, etc. sont le refuge des scènes artistiques off. Techno-travellers, cirque underground, théâtre d'avant-garde, scènes rock, plasticiens, tagueurs, etc. mettent en valeur ces espaces en les transformant en lieux artistiques et culturels off. En investissant des espaces ordinaires ou hors d'usage, les artistes off leur donnent une valeur symbolique portée par l'expérience éphémère qu'ils proposent le temps d'une représentation. Le détournement artistique et l'usage temporaire de l'espace proposent une expérience singulière aux citadins et participent à l'écriture d'un nouveau discours symbolique sur la ville et à la production des représentations sociales de l'espace (Haydn, Temel, 2006). La présence des artistes off est un événement en soi, pour la ville et pour les citadins, l’occasion, rare, de sortir des cadres balisés et routiniers d'une ville de plus en plus standardisées et domestiquées par le cappuccino et la vidéosurveillance (Chatterton, 2002; Atkinson, 2003). Par exemple, le théâtre de rue, dont les initiateurs s'affichaient en opposition avec l'univers théâtral in, transforme et détourne, le temps d'un spectacle, l'espace public en scène de théâtre (Chaudoir, 2000). Ce caractère temporaire de nombreux lieux off participe à leur valorisation symbolique dans le sens où ils deviennent des évènements, des happenings urbains. L'inconstance du off rend sa visite et son expérience plus précieuse : en s’y rendant, le citadin capte l'air du temps, participe à un micro-événement, et pourra dire : «j'étais là ! ». La visite ou la connaissance des milieux off est ainsi un marqueur distinctif pour celui qui sait, qui a connaissance de ce qui se passe dans la cité, de ce qui change, de ce qui est nouveau et éphémère. Par exemple, la scène culturelle off berlinoise est présentée 
par les guides de voyage comme une spécificité locale, produit de l'histoire singulière de la ville. Assister à des événements culturels off s’apparente à la participation à un évènement historique : la réunification et l'invention d'une nouvelle capitale. Le off est un élément de l'expérience touristique et de l'image de la ville. Pour le touriste à Berlin, la visite des lieux et quartiers off s'impose d'autant plus qu'ils seraient sans cesse menacés par la gentrification (Vivant, 2006b).

\section{Les squats d'artistes, ou comment faire du off de nécessité vertu}

Par leur caractère illégal, les squats d'artistes sont une forme idéal-typique des lieux culturels off. Apparus en Europe dans les années 1960 et 1970, dans la foulée des mouvements de contestations politiques et de revendications communautaires, certains de ces lieux sont encore aujourd'hui des lieux alternatifs communautaires, à vocation artistique ou non, comme le quartier Christiania à Copenhague ou les squats des quartiers Kreuzberg et Prenzlauer Berg à Berlin. Dans les années 1980, les premiers squats d'artistes sont ouverts à Paris. Le quartier de Belleville a accueillit plusieurs squats anarchistes autour desquels gravitait la scène rock alternative, dont l'usine Pali Kao, repaires des groupes Béruriers Noirs et Hot Pants, symboles de la culture off punk des années 1980 (Crettiez, Sommier, 2002). Parallèlement, l’autodéfinition de certains squats (par exemple le collectif Art-Cloche) comme étant « artistique » leur a permis de se détacher des autres mouvements de squatters plus politisés et de donner au squat une justification : il ne s’agit plus de révolutionner la société mais seulement la pratique artistique.

Depuis la fin des années 1990, échaudés par des expériences malheureuses, certains squatters choisissent sciemment des localisations plus centrales et symboliques, qui leur offrent une meilleure visibilité dans l'espace urbain, mais aussi dans l'espace médiatique. Par leur positionnement revendicatif et géographique, ces squats d'artistes ont jouit d'une relative notoriété auprès du grand public et d'une attitude conciliante de la part des pouvoirs publics. La principale revendication de ces collectifs d'artistes concerne la pérennisation de l'occupation du bâtiment afin de rendre possible le long et incertain travail de création. Chez Robert Electron Libre est le squat le plus représentatif de cette tendance. Ouvert en novembre 1999 par un groupe d'artistes au 59, rue de Rivoli, situé sur une des principales artères commerçantes de Paris, face à un restaurant McDonald's et à un magasin H\&M, où le $\mathrm{m}^{2}$ commercial est un des plus chers de la capitale, il est devenu la figure de proue de ce mouvement squat parisien ${ }^{3}$. Or si le squat est un

\footnotetext{
${ }^{3}$ Plusieurs autres squats ont mis en œuvre ce type de stratégie comme Socapi en face du musée Picasso ou Bourse, idéalement situé face à la Bourse et à l'Afp. Sur l'évolution des stratégies de localisation et de légitimation des squats d'artistes parisiens, voir : Drouet, 2001; Dorlin-Oberland, 2002; Vivant, 2006b. Remarquons toutefois que l'émergence de squatters stratèges ne s'est pas substituée aux mouvements squats traditionnels : un off du off radicalise le mouvement et les revendications. D'autre part, depuis quelques années, ce mouvement squat, plus discret, est beaucoup moins médiatisé.
} 
moyen de dénoncer le scandale immobilier, la centralité et la visibilité permettent aux artistes de se faire connaître et de vendre leurs œuvres. Ils utilisent sciemment le squat comme un mode d'intégration au monde de l'art, le squat étant un moyen économique d'accéder à un lieu de travail et d'exposition.

En 2001, pendant la campagne électorale municipale, le candidat socialiste, Bertrand Delanoë, a proposé, dans le cadre du volet culturel de son programme électoral, d' «organiser des conventions d'occupation de friches avec des collectifs d'artistes », reprenant une des revendications des artistes squatters. Une fois élu, il a tenu parole et a engagé très rapidement le rachat du 59 rue de Rivoli par la Ville (4,5millions d'euros) en mai 2002 dans le but d'établir une convention d'occupation avec les artistes. Le nouveau propriétaire se trouve confronté à de graves problèmes de sécurité et de remise aux normes du bâtiment, nécessitant des travaux longs et coûteux (estimés à 4,4 millions d'euros) car le bâtiment ayant vocation à accueillir du public, les contraintes de sécurité sont très importantes (Jeanneret, 2004). Comment, par la mobilisation des artistes et la médiatisation des événements artistiques, ces squatters ont-ils su convaincre les édiles parisiens du bien-fondé de leur démarche et de leur nécessaire pérennisation in situ ?

\section{Les Frigos : résistants au cour de Paris Rive Gauche.}

Avec ses façades taguées, ses fêtes et ses événements artistiques, le bâtiment des Frigos, souvent considéré à tort comme un squat, est la figure de proue de la résistance au projet urbain Paris Rive Gauche. Ces artistes off, dont la présence était à l'origine un outil de gestion de la vacance, sont devenus, par leur résistance et leur communication des acteurs incontournables du nouveau quartier.

Le bâtiment des Frigos, construit en 1920 dans le cadre de la réorganisation de l’approvisionnement alimentaire de Paris, a été désaffecté en 1971, suite au transfert du marché alimentaire des Halles à Rungis. En l'absence de projet pour ce site, son propriétaire, la Sncf, n’engagera aucun investissement, tant pour la conservation du bâtiment que pour sa démolition. En 1980, la Sncf, met le bâtiment en location afin d'en retirer de faibles revenus et de contrôler son occupation, autrement dit, d'éviter les squatters ${ }^{4}$. Malgré son manque de confort (absence de sanitaires et de fenêtres), certaines caractéristiques architecturales et techniques du bâtiment (hauteur sous plafond, taille des lots, isolation phonique, forte portance au sol) ainsi que le faible

\footnotetext{
${ }^{4}$ Ce mode de gestion de la vacance par le conventionnement d'occupation temporaire a été utilisé par la Sncf sur d’autres sites comme la Halle Pajol (18 ${ }^{\text {ème }}$ arrondissement). Elle a rencontré les mêmes problèmes de définition des responsabilités (notamment en cas d'accident) et de résistance aux expulsions à l'issue des contrats car les acteurs des scènes artistiques off résistent et s’opposent à l'instrumentalisation dont ils sont l'objet.
} 
montant des loyers satisfaisaient les besoins de certaines activités artistiques et artisanales. Les occupants, titulaires de conventions d'occupation du domaine public, ont réalisé un certain nombre de travaux, parfois importants, pour améliorer le confort et l'habitabilité du bâtiment. Aujourd'hui, le nombre d'usagers du site est estimé à 250 personnes dans 80 ateliers.

Dans le cadre du premier plan d'aménagement de la Zac Paris Rive Gauche adopté en 1991, ce lieu off était menacé de démolition. Evénements artistiques et happenings de résistance urbaine, ont permis aux occupants des Frigos et à d'autres associations du quartier de faire valoir leur vision de l'avenir du quartier, de faire connaitre leurs problèmes, et de faire face à l'aménageur en vue d'influer sur le projet urbain. Progressivement, par sa charge symbolique et médiatique, la destruction des Frigos est devenue impossible et le maintien des Frigos a été acté dans le cadre du plan d'aménagement de Zac modifié en 1996 . La Ville et l'aménageur public (la Semapa) ont ensuite longuement et durement négocié avec les occupants les conditions de cette pérennisation. Deux questions ont été l'objet d'âpres discussions : d'une part, la délimitation du périmètre des Frigos, son environnement immédiat et la création de nouveaux locaux à vocation artisanale et artistique ; d'autre part la question du statut du bâtiment et de ses occupants ainsi que le montant des loyers. Conformément à ses engagements, la Ville de Paris a acheté les Frigos en $2003^{6}$ et a mandaté la Régie Immobilière de la Ville de Paris (Rivp) pour réaliser les travaux de mise en conformité et de sécurité incendie sur le bâtiment ${ }^{7}$. A l'issue de cette cession, les conventions d'occupation ont été transférées, telles quelles, au nouveau propriétaire, mais, les situations des différents occupants n’ont pas été renégociées ni régularisées ${ }^{8}$.

\section{Les nuits off au cour de la ville}

L'organisation d'événements, concerts ou soirées, ancre les lieux off dans le paysage nocturne des villes. La réglementation sur des lieux de loisirs nocturnes in est de plus en plus rigide : limitation des heures d'ouvertures, multiplication des fermetures administratives sur plaintes des riverains, législations sur la consommation d'alcool et de tabac. Ce raidissement s'accompagne d'une autocensure et d'un conformisme croissants des gestionnaires de lieux in : filtrage des entrées et homogénéisation des ambiances (même musique, même décoration, même menu). Dans le même temps, les grandes entreprises transnationales des loisirs et des divertissements jouent un rôle de

\footnotetext{
${ }^{5}$ L'histoire des luttes des occupants des Frigos a été décrite par ailleurs (Vivant, 2006b)

${ }^{6}$ Montant de l'achat : 5,7 millions d'euros (Décision DAUC 20).

${ }^{7}$ Décision du Conseil de Paris du 16 juin 2003, DAC 36. Le coût des travaux est estimé à 1,5 millions d'euros.

${ }^{8}$ La Sncf ayant toujours manifesté sa volonté de céder ce site, elle n’a réalisé aucun investissement, se contentant d’interventions ponctuelles et d'une gestion a minima. En particulier, elle n'a pas effectué les travaux de mise aux normes de sécurité incendie (or deux incendies ont endeuillé les Frigos dans les années 1990) ni cherché à régulariser les situations illégales. Certaines pratiques illicites lors des transferts de baux semblent avoir cours, comme la sous-location non déclarée et le paiement d'un "droit d’entrée ». De plus, si les premiers locataires ont bénéficié de loyers très faibles, le gestionnaire a progressivement réévalué les loyers au fil du temps pour les nouveaux arrivants. Il existe une disparité forte des loyers en fonction de la date d'installation dans les lieux. Les plus anciens et les plus installés (voire, pour les artistes, les plus reconnus) paient des loyers beaucoup plus bas que les locataires les plus récents.
} 
plus en plus important dans la vie nocturne des villes soit en jouant le rôle de sponsor de nombreux événements dont ils orientent les choix artistiques, soit en créant leurs propres espaces (Hard Rock Café, Planet Hollywood, chaîne de discothèques Macumba, club Med World). De véritables empires économiques du monde de la nuit se constituent, comme par exemple au Royaume-Uni où dix entreprises contrôlent la moitié des pubs du pays (Chatterton, 2002). Face à ce formatage des nuits, les lieux off constituent des espaces de liberté et de jeu pour les noctambules. De liberté, car les nuits off sont moins régies par des règles strictes : pas de contrôle à l'entrée, pas d'heure de fermeture, pas de limites de décibels.... De jeu aussi car la recherche de la free party du soir ressemble souvent à un jeu de piste (Chatterton, Hollands, 2003).

Le succès, ou plutôt la survie, des lieux off s’appuie sur une grande diversité de l'offre et de la programmation. Organiser des événements nombreux et éclectiques permet de limiter les risques financiers. Par exemple, en matière musicale, de nombreux groupes s'y produisent, dans des styles plus ou moins confidentiels (de la techno hardcore au garage post-punk). Certains artistes aujourd'hui largement reconnus ont débutés dans ces lieux off, y ont répété, s’y sont produits, y ont construit leur réputation d'artistes off et engagés. Les genres musicaux et artistique présentés dans ces lieux sont porteurs de stéréotypes et de préjugés qui conduisent à imaginer un public plutôt jeune et marginal, ostensiblement en rupture et jouant avec les codes d'appartenances à telle ou telle sous-culture (gothique, hip hop, ...). L’observation apporte un constat plus modéré. Les lieux off attirent des visiteurs très variés. Par exemple, Matjaz Ursic montre que les lieux off de Ljubjana ${ }^{9}$ constituent le troisième pôle d'activité nocturne de la ville et qu'ils sont fréquentés par 20\% des jeunes de 18 à 25ans (Ursic, 2007). De même, par leur localisation stratégique, les squats d'artistes s'inscrivent plus facilement dans une « géographie de la culture » (Libération, 25 avril 2003) et des «sorties branchées » (L’Express, 27 juin 2002) parisiennes. Certains événements deviennent des occasions particulières, d'après la presse, pour s'intéresser à ces lieux. Souvent, des événements off, vernissages d'expositions, concerts ou spectacles, sont signalés au même titre que les événements in dans les pages culturelles des journaux (notamment dans Libération). Par cette médiatisation, les expositions dans les squats d'artistes aiguisent la curiosité d'un public divers, inattendu dans ces lieux, rendus plus accessible par leur localisation géographique. D’ailleurs la presse s'amuse à épingler les stigmates d'appartenance à la bourgeoisie de ces visiteurs occasionnels, comme les «foulards Hermès et les souliers vernis » (Le Point, 25 aout 2000) ou ces « cadres dans une société d'assurance [...] qui profitent de leur pause déjeuner pour découvrir le [squat]. En costume-cravate, ils découvrent le travail de cette horde d'artistes sauvages » (Le Figaro, 10 juin 1999). Ailleurs, de tels codes vestimentaires semblent des stigmates incongrus comme pour ce groupe de jeunes cadres en costume de ville

\footnotetext{
${ }^{9}$ Rog Factory, Orto bar et Metelkova City, occupant d'anciens bâtiments militaires, sont concentrés dans le centre de Ljubljana.
} 
que nous avons vu être refusé à l'entrée d'un club off underground londonien, the Shunt (situé sous la station de métro London Bridge).

Occasionnelle voire unique, la fréquentation des lieux off marque les esprits et les mémoires. Ainsi, dans les années 1980, les Frigos était au cœur d’un no man's land ferroviaire où ont eu lieu des fêtes mémorables, des concerts et divers spectacles, comme les premières représentations du cirque Archaos. Ils sont devenus un lieu symbolique et festif pour toute une génération de jeunes parisiens. Cette réputation se perpétue aujourd'hui par les fêtes et spectacles organisés aux Voutes, squat voisin des Frigos. La réputation d'un lieu off dépasse parfois la communauté de ses usagers et s'inscrit dans l'histoire collective de la vie culturelle et artistique d'une ville. Ainsi, même pour ceux, trop jeunes, qui ne l'ont pas connu, l'Hacienda, club off des années 1980 qui a vu naitre l'essentiel de la scène rock et house britannique, est devenu le symbole de Manchester, mis en valeur aujourd'hui dans une exposition organisée par la ville ${ }^{10}$. Le off d'hier est aujourd'hui in, mis en scène à travers la réécriture de l'histoire culturelle des villes par les institutions locales. Par exemple, le Barbican, un des principaux centre culturel londonien, a accueilli une exposition retraçant les allers-retours entre Londres et New York des artistes punks des années 1975-1985. Ceux qui étaient considérés hier comme des marginaux et des junkies sont présentés aujourd'hui comme des acteurs centraux des scènes artistiques locales, révélant le potentiel et l'histoire créative de ces villes.

Les lieux off appartiennent au monde de la nuit et de la fête, mais certains mettent également en œuvre des activités à destination des populations riveraines : organisation de fêtes de quartier, de brocante et d'ateliers de création (squat Macaq), création d'un espace de gratuité (à la Miroiterie), participation aux opérations portes ouvertes des ateliers d'artistes du quartier.... Ces actions sont autant de moyens de montrer leur bonne volonté aux autorités, de se faire accepter par les riverains, d'être connus par les médias, et ainsi d’obtenir des soutiens plus variés. La pacification des rapports de voisinage est le préalable à toute tentative de discussion et de négociation avec les autorités car sans le soutien des riverains aucun espoir de pérennisation n’est possible.

\section{Les événements off : actes de résistance et de revendications}

L'instabilité d'un squat d'artistes en fait un évènement en soi, que les citadins doivent se hâter d'expérimenter, car sans cesse menacé par une expulsion. En effet, l'événement qui est le plus fréquemment l'objet d'un article est la (menace de) fermeture d'un squat. En mettant en avant le risque d'une expulsion imminente, le journaliste peut susciter la curiosité du lecteur, qui doit se

\footnotetext{
${ }^{10}$ http://www.urbis.org.uk/page.asp?id=3149 (consulté le 19 décembre 2007). Un panneau de cette exposition reprenait les propos de Nick Johnson, urbaniste (agence Urban Splash) et président de Marketing Manchester, qui reconnait n'être jamais allé à l'Hacienda mais qui est fier que ce lieu se situe dans sa ville, Manchester.
} 
dépêcher de venir avant qu'il ne soit trop tard : "faites vite, car le squat de la rue de Rivoli est censé vider les lieux le 4 août. Sauf décision providentielle" (Télérama, 29 juin 2000). Les squatters reconnaissent eux-mêmes utiliser les médias comme une arme car un squat médiatisé permet de « squatter les médias » et de freiner la répression policière (Technikart, mars 2000). Les squatters développent de véritables stratégies de médiatisation mettant en scène le risque d'expulsion et le sursis permanent comme des actes de subversion urbaines et comme des événements. Mais si les médias rendent souvent compte de l'intervention policière pour l'expulsion d'un squat, la plupart des articles font surtout mention d'un risque imminent d'expulsion. Par cette médiatisation, les squatters sensibilisent le public à leurs problèmes et leurs revendications, espérant retarder l'exécution de l'ordre d'expulsion. Ainsi, dès 2000, la presse annonçait que le squat Chez Robert Electron Libre « risque de fermer » (Le Figaro, 25 juillet 2000). Aujourd'hui, la ville de Paris a acheté le bâtiment et doit réaliser des travaux pour pérenniser l'occupation du collectif d'artistes.

D’ailleurs le potentiel médiatique d'un lieu influence les choix de localisation de certains squats d'artistes. S'installer en face de la Bourse de Paris et de l'AFP et à proximité des principales rédactions parisiennes a permis d'accélérer la venue des journalistes, d'établir des contacts et de faire connaître les revendications du squat Bourse. En mettant en avant la dimension artistique de leur occupation, les artistes squatters apportent une forte dimension symbolique à leur action, ce qui leur donne une légitimité plus forte au regard des journalistes. En effet, comme le note Patrick Champagne, « une action symbolique à fort capital culturel tend à être une manifestation « bien vue » (au deux sens) par une partie importante du champ journalistique et a de fortes chances de susciter rapidement la sympathie et la compréhension d'un secteur suffisamment large du champ pour déclencher presque automatiquement une campagne de presse favorable » (Champagne, 1984: 33). Cette forte visibilité dans les médias permet de se protéger contre des expulsions trop musclées, voire de transformer l'expulsion en une sorte de happening politico-artistique. Par exemple, l'expulsion de la Bourse a été mise en scène par les artistes devant les journalistes : les squatters ont eux-mêmes détruit certaines de leurs œuvres, tout en offrant un café et des croissants aux Crs. En invitant les journalistes à assister à l'expulsion, les squatters la publicisent et dramatisent la scène tout en diffusant une image plus positive d'eux-mêmes : conciliants, respectueux et amusants voire sympathiques.

De manière similaire, les événements sont des prétextes à l'exposé des problèmes et des revendications des occupants des Frigos. Ils ont ainsi organisé des actions festives pour protester contre le projet de construction d'un bâtiment entre les Frigos et la Seine. A leurs yeux, ce projet était une double provocation. Mis en œuvre par un groupe financier (Capital et Continental) aux objectifs principalement spéculatifs, ce bâtiment se situe sur une nouvelle parcelle, qui était 
précédemment incluse dans le périmètre «historique » du site des Frigos ${ }^{11}$. La perte de ce territoire avait été vécue comme une traitrise de la part de l'aménageur. La construction d'un bâtiment de bureaux occultant la vue sur la Seine était perçue comme une tentative d'étouffer les Frigos entre le bitume et le béton. Pour informer les parisiens de la situation et prendre à parti les pouvoirs publics, ils ont organisé une fête le 11 novembre 2000, « Ca germe dans le béton », lors de laquelle spectacles, plantation d'arbres et signature de pétitions ont permis d'attirer les médias pour faire valoir leur revendications à savoir le besoin de maintenir leur « espace vital » (pour reprendre leur propres termes) : ce projet étoufferait les Frigos, diminuerait son accessibilité et sa visibilité. Un an plus tard, ils ont récidivé par l'opération « Ca fleurit dans le béton ». Cette mobilisation n’a que partiellement était concluante puisque le bâtiment a été construit, avec toutefois une concession faite aux associations : le percement d'un passage garantissant une vue sur la Seine. Ces événements ont été commentés dans les médias. Cette exposition médiatique participe à la construction de l'image des Frigos comme étant un site d'artistes, fonctionnant comme un collectif, dont les revendications seraient légitimes, face à un aménageur avide et technocratique.

\section{L'événement off comme mode de légitimation du travail artistique}

Un des objectifs du revirement géographique des artistes squatters est la recherche de visibilité et de légitimité du travail et des œuvres des artistes, ainsi que l'accès, par une voie alternative, au marché de l'art, contrôlé par les galeristes, les critiques et les institutions publiques. Le marché de l'art est particulièrement hermétique et rares sont les jeunes artistes qui parviennent à vivre de leur art. Pour eux, le passage en squat peut être une stratégie innovante d'entrée sur le marché de l'art. Si certains squats sont fermés au public pour offrir de meilleures conditions de travail, beaucoup accueillent plus ou moins occasionnellement le public, favorisant les rencontres entre artistes et acheteurs potentiels. La localisation des squats d'artistes dans des quartiers à haute valeur symbolique et à forte concentration de galeries d'art et autres professionnels du monde de l'art, permet la visibilité et la commercialisation de leurs œuvres. Beaucoup de ces squats fonctionnement comme des promoteurs artistiques in en organisant des expositions et leur vernissage, en éditant des flyers d'information sur leurs expositions, en s'intégrant à diverses manifestations artistiques, comme les week-ends " Ateliers ouverts » dans un quartier ou le controversé festival « Art et Squat » organisé par le Palais de Tokyo.

\footnotetext{
${ }^{11}$ Pour reprendre les termes des responsables associatifs qui luttent pour la préservation du site et non pas uniquement du bâtiment.
} 
L’organisation d'évènements particuliers comme les opérations « ateliers ouverts » permet aux artistes d'un quartier d'exposer leurs œuvres, de les faire connaître et de les vendre au sein de leur lieu et environnement de création. Médiatisation de l'œuvre et de l'artiste mais aussi de l'environnement qui a produit cette création, ces opérations « ateliers ouverts » se multiplient dans Paris, les week-ends printaniers étant particulièrement propices à l'organisation de tels événements. Les lieux off participent généralement à ces opérations ; ils profitent ainsi de cette mise en lumière éphémère. La représentation de l'espace de travail et de vie des artistes prend son sens et son ampleur. Elles sont l'occasion pour les citadins de découvertes artistiques, de rencontres avec les artistes, et également de balades originales dans la ville. A travers la presse comme à l'écoute des commentaires des visiteurs, il semble que la découverte du travail des artistes (travaillant en atelier ou en squat) soit éclipsée par le charme de la (re-)découverte d’un quartier et de ses arrières cours ${ }^{12}$.

En 2002, l'ouverture du Palais de Tokyo, nouveau centre de création contemporaine, a suscité une vive indignation des artistes squatters. Ils accusaient l'esthétique intérieure du bâtiment, brute et volontairement inachevée, de singer les squats, comme un simulacre où le in prendrait des airs de off $^{13}$. Sa localisation au cœur du seizième arrondissement et ses possibilités financières (publiques et privées) sont vécues comme des provocations par les squatters qui survivent tous les jours dans la précarité et l’illégalité. Suite à des manifestations lors de son inauguration, la direction du Palais de Tokyo a proposé aux artistes squatters de monter un événement commun, qui prit la forme du festival Art et Squat, en septembre 2002. Lors de ce festival, le Palais de Tokyo jouait le rôle de passeur et de lieu de rencontres où des débats réunissant squatters, professionnels du monde de l'art, chercheurs et représentants d'institutions publiques ont permis de mettre en lumière les spécificités du travail en squat, les difficultés rencontrées par les squatters et les réponses apportées par les pouvoirs publics. Les spectateurs étaient invités à se rendre in situ, dans les squats pour assister à des spectacles, rencontrer les artistes et apprécier leurs conditions d'existence et de travail. A cette fin, une carte localisant l'ensemble des squats participants a été éditée. La presse s'est emparée du festival ; tous les journaux nationaux lui ont consacré un ou plusieurs articles. Mais ensuite, progressivement, les squats d'artistes sont retournés dans l'ombre, dans le off. L’année suivante, l'expérience a été renouvelée, mais le souffle s’est vite épuisé, et le festival n'existe plus aujourd’hui.

\footnotetext{
12 «L'ouverture de deux cent trente ateliers d'artistes conduira les visiteurs dans des cours et des arrière-cours insoupçonnables, à travers des jardinets embaumant le lilas et le chèvrefeuille, sous des vignes même, dans les escaliers cirés d'appartement bourgeoisement tenus, dans un ancien bar reconverti en agence photo, ou sur les allées, entre déchets et récupération, conduisant à la Forge [squattée à l'époque, transformée en ateliers d'artistes depuis], une usine investie il y a près de cinq années, où travaillent une vingtaine de plasticiens ( « Les artistes de Belleville ouvrent leur ateliers », Le Monde, 10 mai 1996).

${ }^{13}$ De nombreux journalistes et observateurs ont souligné et critiqué cette esthétique néo-squat.
} 


\section{Le off au service de la ville créative?}

La présence d'artistes participe à la construction d'une valeur symbolique des espaces, transposée par d'autres en valeur économique (Zukin, 1982, 1995). Même les artistes proposant une alternative au modèle institutionnel de la création, qui pourraient paraître marginaux voire dangereux, sont porteurs de représentations positives et leur présence peut être utilisée à la fois comme un outil de gestion et comme un vecteur d'image. L'évolution de la politique culturelle de la Ville de Paris et l'attitude conciliante de la municipalité vis-à-vis des scènes artistiques off reflètent sa volonté de renforcer et promouvoir toutes les formes de création artistique et illustrent comment les événements off peuvent être utilisés par la Ville pour mettre en scène ou produire l'image d'un projet. Cette mise en scène du off dans le cadre des politiques urbaines et culturelles vise à produire une certaine image de la ville, marquée par les thématique de création et de l'inventivité. Comment la présence d'artistes off, qui véhiculent des représentations singulières de créativité et de transgression, est-elle utilisée aujourd'hui pour animer des quartiers et progressivement en changer l'image ?

\section{Le mythe des Frigos : quand le off donne un semblant de vie à un quartier nouveau}

Sculpteurs, architectes, peintres, photographes, musiciens, artisans travaillent régulièrement aux Frigos. Si une petite galerie a ouvert en 2003, hormis lors des portes ouvertes (deux week-end par an), ils ne sont pas ouvert au public. Pourtant, la présence de ces artistes participe à la construction de l'image des Frigos et du quartier comme nouveau quartier latin. Au gré des événements et au fil des évolutions, achoppements et bagarres de la concertation, les médias ont à plusieurs reprises porté leur attention sur ce site qu'ils ont pris soin de décrire à un public non familier du lieu ${ }^{14}$. Le vocabulaire utilisé dans la presse pour décrire le site fait référence à son passé industriel et à son caractère de friche, tout en lui attribuant un caractère poétique ou en l'esthétisant : « site étrange et poétique », « chaque $\mathrm{cm}^{2}$ est couvert de graphes qui s'entrelacent comme une forêt de lianes où serpentent de gigantesques tuyaux ». Ces descriptions s'appuient sur trois registres sémantiques : la résistance (cuirassier Potemkine, asile éternel, célèbre village gaulois, guérilla), la création et l'art (pépinière de la création contemporaine, lieu décalé, lieu unique, centre artistique, pôle culturel, site de création artistique et artisanale, lieu de culture et de patrimoine, ruche), le monument (adresse mythique, institution, symbole immobilier, lieu

\footnotetext{
${ }^{14}$ Pour connaître l'image des Frigos, nous avons réalisé une revue de presse. Une vingtaine d’articles ont été publiés dans la presse nationale et locale. L'aménageur (Semapa) a consacré plusieurs articles aux Frigos dans son journal, support de communication de l'opération d'aménagement.
} 
emblématique). La dimension artistique des Frigos est mise en lumière par l'exposé des événements (concerts, expositions, portes ouvertes) dans les agendas culturels des médias. Les journalistes évoquent fréquemment les rumeurs et anecdotes concernant les Frigos, construisant progressivement ce qui s’apparente à la légende des Frigos. De nombreux artistes seraient venus, se seraient produits ou auraient débutés aux Frigos : Ray Charles et Dizzie Gillespie seraient venu jouer un soir; les Béruriers Noirs et les Négresses Vertes, figures de proues du punk et du rock alternatif français dans les années 1980 y auraient enregistré leurs premiers albums ; des cinéastes y auraient tourné des films... Ces mythes agissent comme des cautions morales, artistiques et politiques. D’autres rumeurs, légendes et autres histoires obscures et invérifiables circulent : depuis l'organisation de fêtes branchées et la location des locaux à grand prix jusqu'au meurtre d'un résident en passant par les incendies qui ne seraient pas si accidentels... Les mythes et les images produisent du sens autour des Frigos et entretiennent une image à la fois sulfureuse, mystérieuse et poétique.

Ces représentations ont sans doute inspirées l'aménageur qui aujourd'hui met en scène les Frigos dans sa communication. Le journal édité par la Semapa a consacré plusieurs articles aux Frigos. Certains articles exposent les problèmes et propositions en vue de l'intégration des Frigos dans la Zac. Depuis 2002, les articles mettent en lumière les activités artistiques des Frigos et s’appuient sur eux pour développer une image positive et créative du quartier. Le sérieux du travail, l'émulation collective, la créativité sont soulignés par des portraits d'artistes et des photographies de leurs œuvres. Ces descriptions participent à la construction d'une image d'un quartier vivant et dynamique, surtout lorsque la Zac, toujours en travaux, peinait à se représenter comme un lieu de création à Paris ${ }^{15}$. Ainsi alors qu'ils ont longtemps constitué une épine dans le pied de l'aménageur, les Frigos sont mis en avant aujourd'hui pour construire l'image du quartier en tant que nouveau quartier Latin et s’imposent comme élément légitime et qualifiant.

\section{Les événements off : outil de mise en scène des opérations urbaines?}

A Paris et ailleurs, cette logique opportuniste fait progressivement place à une logique de commande publique à visée stratégique. L'occupation de friches par des artistes off s'inscrit dans une commande, dans le cadre d'un programme à plus long terme dont l'objectif est la revalorisation du lieu. Même temporaire, l'occupation d'un lieu par des artistes lui confère une dimension symbolique et une valeur emblématique qui contribueront à la régénération de cet espace. Par exemple, la présence d'un cirque off (le Cirque Electrique) est considérée dès l’origine du projet de création d'un jardin sur la Cour du Maroc (18 ${ }^{\text {ème }}$ arrondissement) comme

\footnotetext{
${ }^{15}$ Aujourd'hui, l'ouverture de l'école d'architecture et de l'université conforte cette image et ce projet.
} 
élément de valorisation et de sécurisation du site et de légitimation de plusieurs opérations d'aménagement. En effet, sur cette ancienne friche ferroviaire, au cœur d'un des quartiers les plus stigmatisés de la capitale (Stalingrad), la Ville a autorisé un cirque off à s’installer le temps des études préparatoires à la création d'un jardin. Pendant un an et demi, de nombreux spectacles et des activités culturelles (bal tango, goûter d’enfants musicaux) ont animés la Cour du Maroc, dont certains ont été inscrits au programme du Festival Paris Quartier d'Eté en 2004, faisant connaître le site au-delà du milieu circassien et attirant un public venu d'autres quartiers parisiens. Le cirque a ainsi permis de désenclaver le site en attirant un public extérieur. Nombreux sont les spectateurs qui, sans le cirque, ne se seraient jamais aventurés dans ce secteur stigmatisé comme dangereux, marqué par la dégradation extrême du bâti, le manque d'entretien des espaces publics, la paupérisation de la population et la cristallisation de la principale scène du trafic et de la consommation de crack. Par ailleurs, la médiatisation de l'expérience vécue par les spectateurs du cirque autant que des spectacles eux-mêmes, a contribué à un changement d'image du site : ce n’est plus un secteur malfamé et infréquentable, mais un lieu «magique », dernier espace de liberté et d'aventure dans la ville. Le coup de projecteur porté sur le secteur par la présence du cirque participe à une stratégie globale de changement d'image du quartier basée à la fois sur des opérations ponctuelles et sur la réalisation d’équipements. Dans ce quartier également, les lieux off inspirent les programmateur d'un nouveau lieu de création artistique municipal, le 104 : ce lieu in se donne, de manière ambigüe, des allures de off ${ }^{16}$.

\section{Le off : espace de liberté en sursis}

Toutefois, ce changement d'attitude des pouvoirs publics vis-à-vis du off ne va pas de soi. Le devenir des lieux off sont toujours subordonnés aux mouvements d'opinions et aux changements politiques. Dans divers pays, des lois restreignant les droits de réunion visent explicitement à interdire certaines activités festives. Les lieux de loisirs nocturnes (in et off) doivent jongler avec des réglementations contraignantes en matière d'horaires, de vente d'alcool, de nuisances sonores, et imposent aux consommateurs leurs propres modes de régulation (comme la sélection à l'entrée). Les nuits off offrent des espaces de liberté sans cesse menacés. Au Royaume Uni, le Criminal Act de 1995 criminalise les pratiques comme les rave party (Chatterton, Hollands, 2003 ; Atkinson, 2003). En France, le projet de loi sur la sécurité intérieure de 2003 envisageait de faciliter les conditions d'expulsion des squatters ${ }^{17}$. La scène techno des raves est l'objet de nombreux dispositifs de surveillance voire de prohibition, entravant son caractère off, sous des

\footnotetext{
${ }^{16}$ Ces exemples ont été décrits par ailleurs (Vivant, sous presse).

${ }^{17}$ La remise en cause du statut des intermittents du spectacle est préjudiciable à la vitalité des petites compagnies et va à l'encontre d'un mouvement international (d'affichage) des politiques publiques en faveur de la création.
} 
prétextes sécuritaires. D’autre part, l'installation d'un lieu off ne se fait pas sans conflits. Les riverains leur reprochent de nombreuses nuisances : le bruit, la saleté, des graffitis et autres formes de marquage territorial, des activités nocturnes,... . La drogue et les attroupements sont les principaux éléments de stigmatisation d'un lieu off et de conflit avec le voisinage. Mais si le lieu est souvent stigmatisé par les discours politiques, peu à peu, la puissance publique peut se l'approprier dans une optique gestionnaire : la territorialisation du trafic de drogues facilite sa gestion sanitaire et policière. Les lieux off sont utilisés comme des moyens de connaissance et de contrôle de pratiques déviantes par les autorités publiques (Raffin, 1998).

De New York à Berlin, d'Amsterdam à Genève, les scènes artistiques off s'imposent comme des acteurs essentiels de la vie culturelle des villes. L'intégration des scènes artistiques off dans les politiques urbaines et la mise en scène des événements off deviennent des outils de promotion de la diversité et de la créativité pour les villes. Cette logique s’inscrit à la suite des travaux sur la ville créative selon lesquels, pour devenir attractives, au-delà de la construction d'infrastructures et des incitations fiscales, les villes doivent s'efforcer de devenir cool en proposant des cafés branchés, des pistes cyclables, des événements culturels, une législation progressiste vis-à-vis des minorités et des scènes off. Si des auteurs comme Richard Florida abusent de la caricature pour proposer leur expertise en matière de diagnostic créatif et si les mêmes promeuvent des actions urbaines tendant à une gentrification généralisée des centres urbains, la question de la créativité demeure un enjeu majeur pour le dynamisme économique et social des villes (Florida, 2002; Vivant, 2006a). Certaines villes, notamment britanniques, jouent pleinement le jeu de la mise en scène de la (supposée) créativité locale comme support de développement économique, notamment par le biais d'agences de développement économique dédiées aux entreprises dites créatives. Londres a par exemple créé Creative London, qui est une branche du London Development Agency, l’agence de développement économique de la ville.

Dans le même temps, certains acteurs des scènes artistiques off se sont appropriés ce discours sur la créativité et la compétition interurbaine, et l'instrumentalisent dans leurs négociations. Ainsi, tout en proposant un mode de vie alternatif et en dénonçant les travers du néolibéralisme global, certains artistes squatters mobilisent les discours actuels sur la créativité comme moteur de développement des villes pour justifier et légitimer leurs pratiques. Par exemple, des squatters d'Amsterdam ont « menacé » de partir s'installer à Rotterdam si la municipalité n’était pas plus compréhensive à leur égard (Uitermark, 2004). De même, à Paris, Yabon, leader de nombreux squats, a ravi les journalistes en déclarant demander « l'asile artistique » à New York. Mais si certains artistes off s'approprient les discours in comme mode de légitimation et d'institutionnalisation, le off demeure par sa dimension temporaire, ludique et festive, une 
expérience extraordinaire et un espace de créativité de liberté et de résistance dans les villes contemporaines. 


\section{Revue de presse indicative}

N'apparaissent ici que les articles cités dans l'article parmi plus de 300 articles référencés et consultés (principalement publiés dans Libération, le Monde et le Parisien, mais aussi les Echos, Télérama, les Inrockuptibles, Le Point, etc.)

Le Figaro, 10 juin 1999, "Les voisins farfelus du Palais Brongniart".

Le Figaro, 25 juillet 2000, "'Nous demandons l'asile artistique"".

Le Figaro, 25 juillet 2000, "Des artistes dans l'engrenage de l'expulsion".

Le Monde, 10 mai 1996, "Les artistes de Belleville ouvrent leurs ateliers".

Le Point, 25août 2000, "Squats d'artistes: les combats de la bohème chic".

L'Express, 27 juin 2002, "Petite géographie des sorties branchées".

Libération, 25 avril 2003, "Dossier spécial : une géographie de la culture. Squats : un défi en règle ».

Technikart, $1^{\mathrm{er}}$ mars 2000, "L'emmerdeur public n"

Télérama, 28juin 2000, "Promenade au squart".

\section{Références}

Ascher, F., 2001, Ces événements nous dépassent, feignons d'en être les organisateurs. Essai sur la société contemporaine, La Tour d'Aigues, Editions de l'Aube.

Ascher, F., 2006, "L'air de la ville rend créatif", Local Contemporain, n².

Atkinson, R., 2003, "Domestication by Cappuccino or a Revenge on Urban Space? Control and Empowerment in the Management of Public Spaces", Urban Studies, vol.40, n9, pp 1829-1943.

Champagne, P., 1984, "La manifestation. La production de l'évènement politique", Actes de la recherche en sciences sociales, $\mathrm{n}^{\circ} 101-102$, pp 40-62.

Chatterton, P., 2002, "'Squatting is Still Legal, Necessary and Free": A Brief Intervention in the Corporate City", Antipode, vol.34, n¹, pp 1-7.

Chatterton, P. and R. Hollands, 2003, Urban Nighscapes. Youth Cultures, Pleasure Spaces and Corporate Power, Londres, Routledge.

Chaudoir, P., 2000, Discours et figures de l'espace public à travers les "arts de la rue". La ville en scènes, Paris, L'Harmattan.

Crettiez, X. and I. Sommier, Eds., 2002, La France rebelle. Tous les foyers, mouvements et acteurs de la contestation, Paris, Editions Michalon.

Donnat, O., 2004, "Les univers culturels des Français", Sociologie et sociétés, vol.36, n¹, pp 87103.

Dorlin-Oberland, F., 2002, Squats d'artistes, perspectives d'un mode d'action. Usages et politiques. Sociologie Politique, Paris, Université Paris 1.

Drouet, F.-X., 2001, Conditions et effets de l'émergence des squats d'artistes parisiens dans la presse écrite. Sciences Politiques, Rennes, Institut d'Etudes Politiques. 
Florida, R. L., 2002, The Rise of the Creative Class : and How it's Transforming Work, Leisure, Community and Everyday Life, New York, NY, Basic Books.

Haydn, F. and R. Temel, Eds., 2006, Temporary Urban Spaces. Concepts for the Use of City Spaces, Basel, Birkhäuser.

Jeanneret, T., 2004, Les squats d'artistes à Paris: quel parti pour la politique municipale? Mastère spécialisé Action Publique, Paris, Ecole Nationale des Ponts et Chaussées.

Lextrait, F., 2001, Friches, laboratoires, fabriques, squats, projets pluridisciplinaires...: une nouvelle époque de l'action culturelle, Paris, Ministère de la Culture, disponible sur Internet: http://www.culture.gouv.fr/culture/actualites/ consulté le 15 janvier 2002.

Masboungi, A. and F. Gravelaine (de), Eds., 2004, Gênes : Penser la ville par les grands événements Paris, Editions de la Villette.

Peterson, R. A., 2004, "Le passage à des goûts omnivores: notions, faits et perspectives", Sociologie et sociétés, vol.36, $\mathrm{n}^{\circ} 1$, pp 145-164.

Raffin, F., 1998, La mise en culture des friches industrielles. Poitiers, Genève, Berlin: de l'épreuve locale au développement de dispositifs transnationaux, Lyon, Ariese, Université Lyon 2, Ministère de l'équipement.

Raffin, F., 2002, Les ritournelles de la culture. De la critique sociale à la participation citoyenne, entre mobilités et ancrages urbains. Ecole Doctorale Sciences Humaines et Sociales, Perpignan, Université de Perpignan.

TransEuropeHalles, Ed., 2001, Les Fabriques. Lieux imprévus, Paris, Editions de l'imprimeur.

Uitermark, J., 2004, "The Co-optation of Squatters in Amsterdam and the Emergence of a Movement Meritocraty: A Critical Reply to Pruijt", International Journal of Urban and Regional Research.

Ursic, M. , 2007, Managing Cultural Diversity in the City. Exclusion/Inclusion of (Sub)Cultures as a Factor for Urban Regeneration. Culture and the City. Future Urban Research in Europe, Kalskrona, European Commission.

Vivant, E., 2006a, "La classe créative existe-t-elle?" Annales de la Recherche Urbaine, n¹01, pp $155-161$.

Vivant, E., 2006b, Le rôle des pratiques culturelles off dans les dynamiques urbaines. Institut Français d'Urbanisme, Champs sur Marne, Université Paris 8.

Vivant, E., sous presse, "Sécurisation, pacification, animation. L'instrumentalisation des scènes culturelles off dans les politiques urbaines", Terrains et travaux. 